\title{
A short survey on pre-Lie algebras
}

\author{
Dominique Manchon*
}

\begin{abstract}
We give an account of fundamental properties of pre-Lie algebras, and provide several examples borrowed from various domains of Mathematics and Physics : Algebra, Combinatorics, Quantum Field Theory and Numerical Analysis.
\end{abstract}

\section{Introduction}

A left pre-Lie algebra over a field $k$ is a $k$-vector space $A$ with a binary composition $\triangleright$ that satisfies the left pre-Lie identity:

$$
(a \triangleright b) \triangleright c-a \triangleright(b \triangleright c)=(b \triangleright a) \triangleright c-b \triangleright(a \triangleright c),
$$

for $a, b, c \in A$. Analogously, a right pre-Lie algebra is a $k$-vector space $A$ with a binary composition $\triangleleft$ that satisfies the right pre-Lie identity:

$$
(a \triangleleft b) \triangleleft c-a \triangleleft(b \triangleleft c)=(a \triangleleft c) \triangleleft b-a \triangleleft(c \triangleleft b) .
$$

The left pre-Lie identity rewrites as:

$$
L_{[a, b]}=\left[L_{a}, L_{b}\right]
$$

where $L_{a}: A \rightarrow A$ is defined by $L_{a} b=a \triangleright b$, and where the bracket on the left-hand side is defined by $[a, b]:=a \triangleright b-b \triangleright a$. As a consequence this bracket satisfies the Jacobi identity.

Pre-Lie algebras are sometimes called Vinberg algebras, as they appear in the work of E. B. Vinberg [29] under the name "left-symmetric algebras" on the classification of homogeneous cones. They appear independently at the same time in the work of M. Gerstenhaber [20] on Hochschild cohomology and deformations of algebras, under the name "pre-Lie algebras" which is now the standard terminology. Note however that Gerstenhaber's pre-Lie algebras live in the category of graded vector spaces, and then additional signs occur in the left pre-Lie identity. The term "chronological algebras" has also been sometimes used, e.g. in the fundamental work of A. Agrachev and R. Gamkrelidze [1].

As we shall see, the notion itself can be however traced back to the work of A. Cayley [5] which, in modern language, describes the pre-Lie algebra morphism $F_{a}$

* The author is grateful to the Erwin Schrödinger Institute (Wien) for warm hospitality during the writing of this paper. 
from the pre-Lie algebra of rooted trees into the pre-Lie algebra of vector fields on $\mathbb{R}^{n}$ sending the one-vertex tree to a given vector field $a$. A very important step has indeed been made in the turn of the century, with the explicit description of free pre-Lie algebras as pre-Lie algebras of decorated rooted trees endowed with grafting. F. Chapoton and M. Livernet [9] obtained this result as a byproduct of their complete description of the operad governing pre-Lie algebras in terms of labelled rooted trees. A. Dzhumadil'daev and C. Löfwall [13] give a more "elementary" proof, avoiding the language of operads, and providing in the same time the explicit description, in terms of rooted trees again, of the free NAP algebra (see below).

An important family of examples of pre-Lie algebras is given by any augmented operad, summing up the partial compositions and quotienting by the actions of the symmetric groups [7], [10]. Another source of examples is given by Rota-Baxter algebras, and more generally by Loday's dendriform algebras and twisted versions of those [14], [18], [16].

The paper is organized as follows: in the first part we recall the main definitions and properties, following [1]. In particular we introduce the group of formal flows of a complete filtered pre-Lie algebra and we prove a pre-Lie version of the PoincaréBirkhoff-Witt theorem. We also recall a recent theorem by J-L. Loday and M. Ronco relating left pre-Lie algebras and right-sided commutative Hopf algebras. The second part is devoted to rooted trees and the free pre-Lie algebra. Following [9] and [7] we recall the construction of the group associated to any augmented operad as well as the associated pre-Lie algebra, and we recall the description of the free pre-Lie algebra in terms of rooted trees. As an application we describe a second pre-Lie algebra product on rooted trees which acts on the first by derivations [4], $[27]$.

The third part is devoted to pre-Lie algebras of vector fields on $\mathbb{R}^{n}$, from the work of A. Cayley [5] to modern developments in numerical analysis through B-series [3], [21], [26], [11].

Finally the last part reviews the relation of pre-Lie algebras with other algebraic structures.

\section{Pre-Lie algebras}

Recall from the introduction that a left pre-Lie algebra over a field $k$ is a $k$-vector space $A$ with a binary composition $\triangleright$ that satisfies the left pre-Lie identity:

$$
(a \triangleright b) \triangleright c-a \triangleright(b \triangleright c)=(b \triangleright a) \triangleright c-b \triangleright(a \triangleright c),
$$

for $a, b, c \in A$. Analogously, a right pre-Lie algebra is a $k$-vector space $A$ with a binary composition $\triangleleft$ that satisfies the right pre-Lie identity:

$$
(a \triangleleft b) \triangleleft c-a \triangleleft(b \triangleleft c)=(a \triangleleft c) \triangleleft b-a \triangleleft(c \triangleleft b) .
$$


As any right pre-Lie algebra $(A, \triangleleft)$ is also a left pre-Lie algebra with product $a \triangleright b:=b \triangleleft a$, we will only consider left pre-Lie algebras for the moment. The left pre-Lie identity rewrites as:

$$
L_{[a, b]}=\left[L_{a}, L_{b}\right]
$$

where $L_{a}: A \rightarrow A$ is defined by $L_{a} b=a \triangleright b$, and where the bracket on the left-hand side is defined by $[a, b]:=a \triangleright b-b \triangleright a$. As a consequence this bracket satisfies the Jacobi identity.

1.1 The group of formal flows. The following is taken from the paper of A. Agrachev and R. Gamkrelidze [1]. Suppose that $A$ is a left pre-Lie algebra endowed with a compatible decreasing filtration, namely $A=A_{1} \supset A_{2} \subset A_{3} \supset \cdots$, such that the intersection of the $A_{j}$ 's reduces to $\{0\}$, and such that $A_{p} \triangleright A_{q} \subset A_{p+q}$. Suppose moreover that $A$ is complete with respect to this filtration. The BakerCampbell-Hausdorff formula:

$$
C(a, b)=a+b+\frac{1}{2}[a, b]+\frac{1}{12}([a,[a, b]]+[b,[b, a]])+\cdots
$$

endows then $A$ with a structure of pro-unipotent group. This group admits a more transparent presentation as follows : introduce a fictitious unit $\mathbf{1}$ such that $\mathbf{1} \triangleright a=a \triangleright \mathbf{1}=a$ for any $a \in A$, and define $W: A \rightarrow A$ by:

$$
W(a):=e^{L_{a}} \mathbf{1}-\mathbf{1}=a+\frac{1}{2} a \triangleright a+\frac{1}{6} a \triangleright(a \triangleright a)+\cdots .
$$

The application $W$ is clearly a bijection. The inverse, denoted by $\Omega$, also appears under the name "pre-Lie Magnus expansion" in [17]. It verifies the equation:

$$
\Omega(a)=\frac{L_{\Omega(a)}}{e^{L_{\Omega(a)}}-I d} a=\sum_{i \geq 0} B_{i} L_{\Omega(a)}^{i} a,
$$

where the $B_{i}$ 's are the Bernoulli numbers. The first few terms are:

$$
\Omega(a)=a-\frac{1}{2} a \triangleright a+\frac{1}{4}(a \triangleright a) \triangleright a+\frac{1}{12} a \triangleright(a \triangleright a)+\cdots
$$

Transferring the $\mathrm{BCH}$ product by means of the map $W$, namely:

$$
a \# b=W(C(\Omega(a), \Omega(b))),
$$

we have $W(a) \# W(b)=W(C(a, b))=e^{L_{a}} e^{L_{b}} \mathbf{1}-\mathbf{1}$, hence $W(a) \# W(b)=W(a)+$ $e^{L_{a}} W(b)$. The product \# is thus given by the simple formula:

$$
a \# b=a+e^{L_{\Omega(a)}} b .
$$


The inverse is given by $a^{\#-1}=W(-\Omega(a))=e^{-L_{\Omega(a)}} \mathbf{1}-\mathbf{1}$. If $(A, \triangleright)$ and $(B, \triangleright)$ are two such pre-Lie algebras and $\psi: A \rightarrow B$ is a filtration-preserving pre-Lie algebra morphism, it is immediate to check that for any $a, b \in A$ we have:

$$
\psi(a \# b)=\psi(a) \# \psi(b) .
$$

In other words, the group of formal flows is a functor from the category of complete filtered pre-Lie algebras to the category of groups.

When the pre-Lie product $\triangleright$ is associative, all this simplifies to:

$$
a \# b=a \triangleright b+a+b
$$

and

$$
a^{\#-1}=\frac{1}{1+a}-1=\sum_{n \geq 1}(-1)^{n} a_{n}
$$

\subsection{The pre-Lie Poincaré-Birkhoff-Witt theorem.}

Theorem 1.1. Let $A$ be any left pre-Lie algebra, $S(A)$ its symmetric algebra, i.e. the free commutative algebra on $A$, and let $\mathcal{U}(A)$ the enveloping algebra of the underlying Lie algebra $A_{\text {Lie }}$, endowed with its usual increasing filtration. There exists a left $\mathcal{U}(A)$-module structure on $S(A)$ and a canonical left $\mathcal{U}(A)$-module isomorphism $\eta: \mathcal{U}(A) \rightarrow \mathcal{S}(A)$, such that the associated graded linear map $\operatorname{Gr} \eta$ : $\operatorname{Gr} \mathcal{U}(A) \rightarrow S(A)$ is an isomorphism of commutative graded algebras.

Proof. The Lie algebra morphism

$$
\begin{aligned}
L: A & \longrightarrow \text { End } A \\
a & \longmapsto\left(L_{a}: b \longmapsto a \triangleright b\right)
\end{aligned}
$$

extends by Leibniz rule to a unique Lie algebra morphism $L: A \rightarrow \operatorname{Der} S(A)$. Now we claim that the map $M: A \rightarrow$ End $S(A)$ defined by:

$$
M_{a} u=a u+L_{a} u
$$

is a Lie algebra morphism. Indeed we have for any $a, b \in A$ and $u \in S(A)$ :

$$
\begin{aligned}
M_{a} M_{b} u & =M_{a}\left(b u+L_{b} u\right) \\
& =a b u+a L_{b} u+L_{a}(b u)+L_{a} L_{b} u \\
& =a b u+a L_{b} u+b L_{a} u+(a \triangleright b) u+L_{a} L_{b} u .
\end{aligned}
$$

Hence

$$
\begin{aligned}
{\left[M_{a}, M_{b}\right] u } & =(a \triangleright b-b \triangleright a) u+\left[L_{a}, L_{b}\right] u \\
& =M_{[a, b]} u
\end{aligned}
$$


which proves the claim. Now $M$ extends, by universal property of the enveloping algebra, to a unique algebra morphism $M: \mathcal{U}(A) \rightarrow$ End $S(A)$. The linear map:

$$
\begin{aligned}
\eta: \mathcal{U}(A) & \longrightarrow S(A) \\
u & \longmapsto M_{u} \cdot 1
\end{aligned}
$$

is clearly a morphism of left $\mathcal{U}(A)$-modules. It is immediately seen by induction that for any $a_{1}, \ldots, a_{n} \in A$ we have $\eta\left(a_{1} \cdots a_{n}\right)=a_{1} \cdots a_{n}+v$ where $v$ is a sum of terms of degree $\leq n-1$. This proves the theorem.

Let us recall that the symmetrization map $\sigma: \mathcal{U}(A) \rightarrow S(A)$, uniquely determined by $\sigma\left(a^{n}\right)=a^{n}$ for any $a \in A$ and any integer $n$, is an isomorphism for the two $A_{\mathrm{Lie}}$-module structures given by the adjoint action. This is not the case for the map $\eta$ defined above. Let us finally notice that, if $p$ stands for the projection from $S(A)$ onto $A$, we easily get for any $a_{1}, \ldots, a_{k} \in A$ :

$$
p \circ \eta\left(a_{1} \cdots a_{k}\right)=L_{a_{1}} \cdots L_{a_{k}} \mathbf{1}=a_{1} \triangleright\left(a_{2} \triangleright\left(\cdots\left(a_{k-1} \triangleright a_{k}\right) \ldots\right)\right)
$$

by a simple induction on $k$. The linear isomorphism $\eta$ transfers the product of the enveloping algebra $\mathcal{U}(A)$ into a noncommutative product $*$ on $\mathcal{S}(A)$ defined by:

$$
s * t=\eta\left(\eta^{-1}(s) \eta^{-1}(t)\right) .
$$

Suppose now that $A$ is endowed with a complete decreasing compatible filtration as is Paragraph 1.1. This filtration induces a complete decreasing filtration $S(A)=S(A)_{0} \supset S(A)_{1} \supset S(A)_{2} \supset \cdots$, and the product $*$ readily extends to the completion $\widehat{S}(A)$. For any $a \in A$, the application of (17) gives:

$$
p\left(e^{* a}\right)=W(a)
$$

as an equality in the completed symmetric algebra $\widehat{S}(A)$.

According to (11) we can identify the pro-unipotent group $\left\{e^{* a}, a \in A\right\} \subset \widehat{S}(A)$ and the group of formal flows of the pre-Lie algebra $A$ by means of the projection $p$, namely:

$$
p\left(e^{* a}\right) \# p\left(e^{* b}\right)=p\left(e^{* a} * e^{* b}\right)
$$

for any $a, b \in A$.

1.3 Right-sided commutative Hopf algebras and the Loday-Ronco theorem. Let $\mathcal{H}$ be a commutative Hopf algebra. Following [24], we say that $\mathcal{H}$ is right-sided if it is free as a commutative algebra, i.e. $\mathcal{H}=S(V)$ for some $k$-vector space $V$, and if the coproduct verifies :

$$
\Delta(V) \subset \mathcal{H} \otimes V .
$$


Suppose moreover that $V=\bigoplus_{n \geq 0}$ is graded with finite-dimensional homogeneous components. Then the graded dual $A=V^{0}$ is a left pre-Lie algebra, and by the Milnor-Moore theorem, the graded dual $\mathcal{H}^{0}$ is isomorphic to the enveloping algebra $\mathcal{U}\left(A_{\text {Lie }}\right)$ as graded Hopf algebra. Conversely, for any graded pre-Lie algebra $A$ the graded dual $\mathcal{U}\left(A_{\mathrm{Lie}}\right)^{0}$ is free commutative right-sided ([24] Theorem 5.3).

\section{Operads, pre-Lie algebras and rooted trees}

We first associate a (right) pre-Lie algebra to any augmented operad, following $\mathrm{F}$. Chapoton [7], and then we recall the description of the Pre-Lie operad itself by F. Chapoton and M. Livernet [9], thus leading to two pre-Lie structures on the vector space generated by the rooted trees.

2.1 Pre-Lie algebras associated to augmented operads. Recall that an augmented operad $\mathcal{P}$ (in the symmetric monoidal category of vector spaces on some field $k$ ) is given by a collection of vector spaces $\left(\mathcal{P}_{n}\right)_{n \geq 1}$ with $\mathcal{P}_{1}=k . e$, an action of the symmetric group $S_{n}$ on $\mathcal{P}_{n}$, and a collection of partial compositions:

$$
\begin{aligned}
\circ_{i}: \mathcal{P}_{k} \otimes \mathcal{P}_{l} & \longrightarrow \mathcal{P}_{k+l-1}, \quad i=1, \ldots, k \\
(a, b) & \longmapsto a \circ_{i} b
\end{aligned}
$$

which, for any $a \in \mathcal{P}_{k}, b \in \mathcal{P}_{l}, a \in \mathcal{P}_{m}$ and $i \in\{1, \ldots, k\}, j \in\{1, \ldots, l\}$, satisfies the associativity conditions:

$$
\begin{array}{rr}
\left(a \circ_{i} b\right) \circ_{i+j-1} c=a \circ_{i}\left(b \circ_{j} c\right), & i \in\{1, \ldots, k\}, j \in\{1, \ldots, l\}, \\
\left(a \circ_{i} b\right) \circ_{l+j-1} c=\left(a \circ_{j} c\right) \circ_{i} b, & i, j \in\{1, \ldots, k\}, i<j,
\end{array}
$$

the unit axiom:

$$
\begin{aligned}
e \circ a & =a \\
a \circ_{i} e & =a, \quad i=1, \ldots, k,
\end{aligned}
$$

as well as the following equivariance condition:

$$
\sigma(a) \circ_{\sigma_{i}} \tau(b)=\iota_{i}(\sigma, \tau)\left(a \circ_{i} b\right)
$$

where $\iota_{i}(\sigma, \tau) \in S_{k+l-1}$ is defined by letting $\tau$ permute the set $E_{i}=\{i, i+1, \ldots, i+$ $l-1\}$ of cardinality $l$, and then by letting $\sigma$ permute the set $\left\{1, \ldots, i-1, E_{i}, i+\right.$ $l, \ldots, k+l-1\}$ of cardinality $k$. The global composition is defined by:

$$
\begin{aligned}
\gamma: \mathcal{P}_{n} \otimes \mathcal{P}_{k_{1}} \otimes \cdots \otimes \mathcal{P}_{k_{n}} & \longrightarrow \mathcal{P}_{k_{1}+\cdots+k_{n}} \\
\left(a, b_{1}, \ldots, b_{n}\right) & \longmapsto\left(\ldots\left(\left(a \circ_{n} b_{n}\right) \circ_{n-1} b_{n-1}\right) \cdots\right) \circ_{1} b_{1} .
\end{aligned}
$$


The free $\mathcal{O}$-algebra with one generator is given by:

$$
F_{\mathcal{P}}: \bigoplus_{n \geq 1} \mathcal{P}_{n} / S_{n}
$$

The sum of the partial compositions yields a right pre-Lie algebra structure on $F_{\mathcal{P}}^{+}:=\bigoplus_{n \geq 2} \mathcal{P}_{n} / S_{n}$, namely:

$$
\bar{a} \triangleleft \bar{b}:=\sum_{i=1}^{k} \overline{a \circ_{i} b} .
$$

Following F. Chapoton [7] one can consider the pro-unipotent group $G_{\mathcal{P}}^{e}$ associated with the completion of the pre-Lie algebra $F_{\mathcal{P}}^{+}$for the filtration induced by the grading. More precisely Chapoton's group $G_{\mathcal{P}}$ is given by the elements $g \in \widehat{F_{\mathcal{P}}}$ such that $g_{1} \neq 0$, whereas $G_{\mathcal{P}}^{e}$ is the subgroup of $G_{\mathcal{P}}$ formed by elements $g$ such that $g_{1}=e$.

Any element $a \in \mathcal{P}_{n}$ gives rise through $\gamma$ to a $n$-ary operation $F_{a}: F_{\mathcal{P}}^{\otimes n} \rightarrow F_{\mathcal{P}}$, and for any $x, y_{1}, \ldots, y_{n} \in F_{\mathcal{P}}^{+}$we have ${ }^{1}[27]$ :

$$
F_{a}\left(y_{1}, \ldots, y_{n}\right) \triangleleft x=\sum_{j=1}^{n} F_{a}\left(y_{1}, \ldots, y_{j} \triangleleft x, \ldots, y_{n}\right) .
$$

2.2 The Pre-Lie operad. Recall that for any operad $\mathcal{O}$, an $\mathcal{O}$-algebra is a vector space $V$ together with a morphism of operads from $\mathcal{O}$ to the operad $\operatorname{Endop}(V)$, where $\operatorname{Endop}(V)_{n}:=\mathcal{L}\left(V^{\otimes n}, V\right)$ with obvious symmetric group actions and compositions. In this sense, pre-Lie algebras are algebras over the pre-Lie operad, which has been described in detail by F. Chapoton and M. Livernet in [9] as follows: $\mathcal{P} \mathcal{L}_{n}$ is the vector space of labelled rooted trees, and partial composition $s \circ_{i} t$ is given by summing all the possible ways of inserting the tree $t$ inside the tree $s$ at the vertex labelled by $i$.

The free right pre-Lie algebra with one generator is then given by the space $\mathcal{T}=\bigoplus_{n \geq 1} T_{n}$ of rooted trees, as quotienting with the symmetric group actions amounts to neglect the labels. The pre-Lie operation $(s, t) \mapsto(s \leftarrow t)$ is given by the sum of the graftings of $t$ on $s$ at all vertices of $s$. As a consequence of (29) we have two pre-Lie operations on $\mathcal{T}^{\prime}=\bigoplus_{n \geq 2} T_{n}$ which interact as follows [27]:

$$
(s \leftarrow t) \triangleleft u=(s \triangleleft u) \leftarrow t+s \leftarrow(t \triangleleft u) .
$$

The first pre-Lie operation $\triangleleft$ comes from the fact that $\mathcal{P} \mathcal{L}$ is an augmented operad, whereas the second pre-Lie operation $\leftarrow$ comes from the fact that $\mathcal{P} \mathcal{L}$ is the pre-Lie operad itself!

\footnotetext{
${ }^{1}$ We thank Muriel Livernet for having brought this point to our attention.
} 
2.3 Two Hopf algebras of rooted forests. Let us consider the two commutative Hopf algebras $\mathcal{H}_{C K}$ and $\mathcal{H}$ associated respectively to the left pre-Lie algebras $(\mathcal{T}, \rightarrow)$ and $\left(\mathcal{T}^{\prime}, \triangleright\right)$ by the functor described in Paragraph 1.3. The first is the Connes-Kreimer Hopf algebra of rooted forests [12]. Its coproduct is described in terms of admissible cuts [19], or alternatively as follows [26, Paragraph 3.2 and Section 7]: the set $U$ of vertices of a forest $u$ is endowed with a partial order defined by $x \leq y$ if and only if there is a path from a root to $y$ passing through $x$. Any subset $W$ of the set of vertices $U$ of $u$ defines a subforest $w$ of $u$ in an obvious manner, i.e. by keeping the edges of $u$ which link two elements of $W$. The coproduct is then defined by:

$$
\Delta_{\mathrm{CK}}(u)=\sum_{\substack{V \amalg W=U \\ W<V}} v \otimes w .
$$

Here the notation $V<W$ means that $x<y$ for any vertex $x$ of $v$ and any vertex $y$ of $w$ such that $x$ and $y$ are comparable. The Hopf algebra $\mathcal{H}_{C K}$ is graded by the number of vertices.

The coproduct on the second Hopf algebra $\mathcal{H}$ is defined as follows: one identifies the unit of $S\left(\mathcal{T}^{\prime}\right)$ with the rooted tree $\bullet$. A subforest of a tree $t$ is either the trivial forest $\bullet$, or a collection $\left(t_{1}, \ldots, t_{n}\right)$ of pairwise disjoint subtrees of $t$, each of them containing at least one edge. In particular two subtrees of a subforest cannot have any common vertex.

Let $s$ be a subforest of a rooted tree $t$. Denote by $t / s$ the tree obtained by contracting each connected component of $s$ onto a vertex. We turn $\mathcal{H}$ into a bialgebra by defining a coproduct $\Delta: \mathcal{H} \rightarrow \mathcal{H} \otimes \mathcal{H}$ on each tree $t \in \mathcal{T}^{\prime}$ by :

$$
\Delta(t)=\sum_{s \subseteq t} s \otimes t / s
$$

where the sum runs over all possible subforests (including the unit and the full subforest $t$ ). As usual we extend the coproduct $\Delta$ multiplicatively onto $S\left(\mathcal{T}^{\prime}\right)$. This makes $\mathcal{H}:=\bigoplus_{n>0} \mathcal{H}_{n}$ a connected graded Hopf algebra, where the grading is defined by the number of edges.

It turns out that $\mathcal{H}_{C K}$ is left comodule coalgebra over $\mathcal{H}$ [4]. The coaction $\Phi: \mathcal{H}_{C K} \rightarrow \mathcal{H} \otimes \mathcal{H}_{C K}$ is the algebra morphism given by $\Phi(\mathbf{1})=\bullet \otimes \mathbf{1}$ and $\phi(t)=\Delta_{\mathcal{H}}(t)$ for any nonempty tree $t$

\section{Pre-Lie algebras of vector fields}

3.1 Flat torsion-free connections. Let $M$ be a differentiable manifold, and let $\nabla$ the covariant derivation operator associated to a connection on the tangent 
bundle $T M$. The covariant derivation is a bilinear operator on vector fields (i.e. two sections of the tangent bundle): $(X, Y) \mapsto \nabla_{X} Y$ such that the following axioms are fulfilled:

$$
\begin{aligned}
\nabla_{f X} Y & =f \nabla_{X} Y \\
\nabla_{X}(f Y) & =f \nabla_{X} Y+(X . f) Y \text { (Leibniz rule). }
\end{aligned}
$$

The torsion of the connection $\tau$ is defined by:

$$
\tau(X, Y)=\nabla_{X} Y-\nabla_{Y} X-[X, Y]
$$

and the curvature tensor is defined by:

$$
R(X, Y)=\left[\nabla_{X}, \nabla_{Y}\right]-\nabla_{[X, Y]} .
$$

The connection is flat if the curvature $R$ vanishes identically, and torsion-free if $\tau=0$. The following crucial observation is an immediate consequence of (3):

Proposition 3.1. For any smooth manifold $M$ endowed with a flat torsion-free connection $\nabla$, the space $\chi(M)$ of vector fields is a left pre-Lie algebra, with pre-Lie product given by:

$$
X \triangleright Y:=\nabla_{X} Y
$$

Note that on $M=\mathbb{R}^{n}$ endowed with its canonical flat torsion-free connection, the pre-Lie product is given by:

$$
\left(f_{i} \partial_{i}\right) \triangleright\left(f_{j} \partial_{j}\right)=f_{i}\left(\partial_{i} f_{j}\right) \partial_{j} .
$$

3.2 Pre-history of pre-Lie: the work of A. Cayley. As early as 1857, A. Cayley [5] discovered a link between rooted trees and vector fields on the manifold $\mathbb{R}^{n}$ endowed with its natural flat torsion free connection. A vector field, which writes:

$$
X=\sum_{i=1}^{n} f_{i} \partial_{i},
$$

is seen as an operandator, since it mixes partial derivation operators $\partial_{i}$ and operands (i.e. functions) $f_{i}$. He remarks (with our modern notations) that for two vector fields $X$ and $Y$, the operator $X \circ Y-\nabla_{X} Y$ is symmetric in $X$ and $Y$ and calls it the "algebraic product" $X \times Y$ of $X$ and $Y$. It is not difficult to check that for any $f \in C^{\infty}$ we have:

$$
(X \times Y)(f)=X .(Y . f)-\left(\nabla_{X} Y\right) . f=f^{\prime \prime}(X, Y),
$$

where $f^{\prime \prime}$ is the second differential of $f$. This still makes sense if $f$ is replaced by a third vector field $Z$, the result being a vector field. Then for any rooted tree $t$ with $n$ vertices, each vertex $v$ being decorated by a vector field $X_{v}$, he defines a new vector field $\mathcal{Y}(t)$. We can understand the construction of $\mathcal{Y}(t)$ by 
the following recursive procedure [21]: the decorated tree $t$ is obtained by grafing all its branches $t_{k}$ on the root $r$ decorated by the vector field $X_{r}=\sum_{i=1}^{n} f_{i} \partial_{i}$, i.e. it writes $B_{+}^{X_{r}}\left(t_{1}, \ldots, t_{k}\right)$. Hence we set:

$$
\begin{aligned}
\mathcal{Y}\left(\bullet_{X_{r}}\right) & =X_{r} \\
\mathcal{Y}(t) & =\sum_{i=1}^{n} f_{i}(t) \partial_{i} \text { with : } \\
f_{i}(t) & =f_{i}^{(k)}\left(\mathcal{Y}\left(t_{1}\right), \ldots, \mathcal{Y}\left(t_{n}\right)\right) .
\end{aligned}
$$

3.3 Relating two pre-Lie structures. Keeping the notations of the previous paragraph, consider the left grafting $\rightarrow$ of two decorated trees, namely:

$$
s \rightarrow t=\sum_{v \text { vertex of } t} s \rightarrow_{i} v .
$$

Considering the Cayley map $t \mapsto Y(t)$ from vector field-decorated rooted trees to vector field, it can be shown, as a direct consequence of the Leibniz rule, that:

$$
Y(s \rightarrow t)=Y(s) \triangleright Y(t) .
$$

In other words, the Cayley map $\mathcal{Y}$ is a pre-Lie algebra morphism. Now consider the map $d_{X}$ from rooted trees to vector field-decorated rooted trees, which decorates each vertex by $X$. It is obviously a pre-Lie algebra morphism, and $F_{X}:=\mathcal{Y} \circ d_{X}$ is the unique pre-Lie algebra morphism which sends the one-vertex tree $\bullet$ to the vector field $X$. The uniqueness of course comes from the fact that the span of rooted trees with left grafting is the free left pre-Lie algebra with one generator $([9],[13])$.

3.4 B-series, composition and substitution. Consider any left pre-Lie algebra $(A, \triangleright)$, introduce a fictitious unit $\mathbf{1}$ such that $\mathbf{1} \triangleright a=a \triangleright \mathbf{1}=a$ for any $a \in A$, and consider for any $a \in A$ the unique left pre-Lie algebra morphism $F_{a}:(\mathcal{T}, \longrightarrow) \longrightarrow(A, \triangleright)$ such that $F_{a}(\bullet)=a$. A $B$-series is an element of $h A[[h]] \oplus k .1$ defined by:

$$
B(\alpha ; a):=\alpha(\emptyset) \mathbf{1}+\sum_{s \in T} h^{v(s)} \frac{\alpha(s)}{\sigma(s)} F_{a}(s),
$$

where $\alpha$ is any linear form on $\mathcal{T} \oplus k \emptyset$. It matches the usual notion of $B$-series [21] when $A$ is the pre-Lie algebra of vector fields on $\mathbb{R}^{n}$ defined at the beginning of the section (it is also convenient to set $F_{a}(\emptyset)=\mathbf{1}$ ). In this case, the vector fields $F_{a}(t)$ for a tree $t$ are differentiable maps from $\mathbb{R}^{n}$ to $\mathbb{R}^{n}$ called elementary differentials. $B$-series can be composed coefficientwise, as series in the indeterminate $h$ whose coefficients are maps from $\mathbb{R}^{n}$ to $\mathbb{R}^{n}$. The same definition with trees decorated by 
a set of colours $\mathcal{D}$ leads to straightforward generalizations. For example $P$-series used in partitioned Runge-Kutta methods [21] correspond to bi-coloured trees.

A slightly different way of defining $B$-series is the following: consider the unique pre-Lie algebra morphism $\mathcal{F}_{a}: \mathcal{T} \rightarrow h A[h]$ such that $\mathcal{F}_{a}(\bullet)=h a$. It respects the graduations given by the number of vertices and the powers of $h$ respectively, hence it extends to $\mathcal{F}_{a}: \widehat{\mathcal{T}} \rightarrow h A[[h]]$, where $\widehat{\mathcal{T}}$ is the completion of $\mathcal{T}$ with respect to the graduation. We further extend it to the empty tree by setting $\mathcal{F}_{a}(\emptyset)=\mathbf{1}$. We have then:

$$
B(\alpha ; a)=\mathcal{F}_{a} \circ \delta^{-1}(\alpha),
$$

where $\delta$ is the isomorphism from $\widehat{\mathcal{T}} \oplus k \emptyset$ to $(\mathcal{T} \oplus k \emptyset)^{*}$ given by the normalized dual basis.

We restrict ourselves to $B$-series $B(\alpha ; a)$ with $\alpha(\emptyset)=1$. Such $\alpha$ 's are in oneto-one correspondence with characters of the algebra of forests (which is the underlying algebra of $\mathcal{H}_{C K}$ ) by setting:

$$
\alpha\left(t_{1} \cdots t_{k}\right):=\alpha\left(t_{1}\right) \cdots \alpha\left(t_{k}\right) .
$$

The Hairer-Wanner theorem [21, Theorem III.1.10] says that composition of $B$ series corresponds to the convolution product of characters of $\mathcal{H}_{C K}$, namely:

$$
B(\beta ; a) \circ B(\alpha ; a)=B(\alpha * \beta, a),
$$

where linear forms $\alpha, \beta$ on $\mathcal{T} \oplus k \emptyset$ and their character counterparts are identified modulo the above correspondence.

Let us now turn to substitution, after [11]. The idea is to replace the vector field $a$ in a $B$-series $B(\beta ; a)$ by another vector field $a$ which expresses itself as a $B$-series, i.e. $a=h^{-1} B(\alpha ; a)$ where $\alpha$ is now a linear form on $\mathcal{T} \oplus k \emptyset$ such that $\alpha(\emptyset)=0$. We suppose here moreover that $\alpha(\bullet)=1$. Such $\alpha$ 's are in one-to-one correspondence with characters of $\mathcal{H}$. The following proposition is proved in [4]:

Proposition 3.2. For any linear forms $\alpha, \beta$ on $\mathcal{T}$ with $\alpha(\bullet=1)$, we have:

$$
B\left(\beta ; \frac{1}{h} B(\alpha ; a)\right)=B(\alpha \star \beta ; a),
$$

where $\alpha$ is multiplicatively extended to forests, $\beta$ is seen as an infinitesimal character of $\mathcal{H}_{C K}$ and where $\star$ is the dualization of the left coaction of $\mathcal{H}$ on $\mathcal{H}_{C K}$.

The condition $\alpha(\bullet)$ is in fact dropped in [4, Propsition 15]: the price to pay is replacing the Hopf algebra $\mathcal{H}$ by a non-connected bialgebra $\widetilde{\mathcal{H}}=S(\mathcal{T})$ with a suitable coproduct, such that $\mathcal{H}$ is obtained as the quotient $\widetilde{\mathcal{H}} / \mathcal{J}$, where $\mathcal{J}$ is the ideal generated by $\bullet-\mathbf{1}$. The substitution product $\star$ then coincides with the one considered in [11] via natural identifications. 


\section{Other algebraic structures}

A dendriform algebra [23] over the field $k$ is a $k$-vector space $A$ endowed with two bilinear operations, denoted $\prec$ and $\succ$ and called right and left products, respectively, subject to the three axioms below:

$$
\begin{aligned}
& (a \prec b) \prec c=a \prec(b \prec c+b \succ c) \\
& (a \succ b) \prec c=a \succ(b \prec c) \\
& a \succ(b \succ c)=(a \prec b+a \succ b) \succ c .
\end{aligned}
$$

One readily verifies that these relations yield associativity for the product

$$
a * b:=a \prec b+a \succ b .
$$

However, at the same time the dendriform relations imply that the bilinear products $\triangleright$ and $\triangleleft$ defined by:

$$
a \triangleright b:=a \succ b-b \prec a, \quad a \triangleleft b:=a \prec b-b \succ a,
$$

are left pre-Lie and right pre-Lie respectively. The associative operation $*$ and the pre-Lie operations $\triangleright, \triangleleft$ all define the same Lie bracket:

$$
\llbracket a, b \rrbracket:=a * b-b * a=a \triangleright b-b \triangleright a=a \triangleleft b-b \triangleleft a .
$$

Observe that:

$$
a * b+b \triangleright a=a \succ b+b \succ a .
$$

This identity generalizes to any number of elements, expressing the symmetrization of $\left(\ldots\left(\left(a_{1} \succ a_{2}\right) \succ a_{3}\right) \cdots\right) \succ a_{n}$ in terms of the associative product and the left pre-Lie product [18]. We stress here that in the commutative case (commutative dendriform algebras are also named Zinbiel algebras [22], [23]), the left and right operations are further required to identify, so that $a \succ b=b \prec a$. In this case both pre-Lie products vanish. A natural example of a commutative dendriform algebra is given by the shuffle algebra in terms of half-shuffles [28]. Any associative algebra $A$ equipped with a linear integral like map $I: A \rightarrow A$ satisfying the integration by parts rule also gives a dendriform algebra, when $a \prec b:=a I(b)$ and $a \succ b:=I(a) b$. The left pre-Lie product is then given by $a \triangleright b=[I(a), b]$.

\section{References}

[1] A. Agrachev, R. Gamkrelidze, Chronological algebras and nonstationary vector fields, J. Sov. Math. 17 No1 (1981), 1650-1675.

[2] Ch. Brouder, Runge-Kutta methods and renormalization, Europ. Phys. J. C12, (2000) 512-534.

[3] J.C. Butcher, Coefficients for the study of Runge-Kutta integration processes, J. Austral. Math. Soc. 3 (1963), 185-201. 
[4] D. Calaque, K. Ebrahimi-Fard, D. Manchon, Two interacting Hopf algebras of trees, Adv. Appl. Math. (to appear). arXiv:0806.2238v2 [math.CO].

[5] A. Cayley, On the theory of the analytical forms called trees, Phil. Mag. 13 (1857), $172-176$.

[6] F. Chapoton, Algèbres pré-Lie et algèbres de Hopf liées à la renormalisation, ComptesRendus Acad. Sci., 332 Série I (2001), 681-684.

[7] F. Chapoton, Rooted trees and an exponential-like series, preprint arXiv:math/0209104 (2002).

[8] F. Chapoton, Un théorème de Cartier-Milnor-Moore-Quillen pour les bigèbres dendriformes et les algèbres braces, Journal of Pure and Applied Algebra, 168 No 1 (2002), $1-18$.

[9] F. Chapoton, M. Livernet, Pre-Lie algebras and the rooted trees operad, Int. Math. Res. Not. 2001 (2001), 395-408.

[10] F. Chapoton, M. Livernet, Relating two Hopf algebras built from an operad, Int. Math. Res. Not., published online, doi:10.1093/imrn/rnm131 (2007).

[11] Ph. Chartier, E. Hairer, G. Vilmart, Numerical integrators based on modified differential equations, Math. Comp., 76 (2007), 1941-1953.

[12] A. Connes, D. Kreimer, Hopf algebras, Renormalization and Noncommutative Geometry, Comm. in Math. Phys. 199 (1998), 203-242.

[13] A. Dzhumadil'daev, C. Löfwall, Trees, free right-symmetric algebras, free Novikov algebras and identities, Homology, Homotopy and Appl. 4 No. 2 (2002), 165-190.

[14] K. Ebrahimi-Fard, Loday-type algebras and the Rota-Baxter relation, Letters in Mathematical Physics 61 (2002), 139-147.

[15] K. Ebrahimi-Fard, D. Manchon and F. Patras, New identities in dendriform algebras, Journal of Algebra 320 (2008), 708-727.

[16] K. Ebrahimi-Fard, D. Manchon, Twisted dendriform algebras and the pre-Lie Magnus expansion, Preprint arXiv:math/0910.2166 (2009).

[17] K. Ebrahimi-Fard, D. Manchon, A Magnus- and Fer-type formula in dendriform algebras, Found. of Comput. Math. 9 No.3 (2009).

[18] K. Ebrahimi-Fard, D. Manchon, F. Patras, New identities in dendriform algebras, Journal of Algebra 320 (2008), 708-727.

[19] L. Foissy, Les algèbres de Hopf des arbres enracinés décorés I + II, PhD Thesis, Univ. de Reims (2002), and Bull. Sci. Math. 126, No. 3, 193-239 and No 4, 249-288 (2002).

[20] M. Gerstenhaber, The cohomology structure of an associative ring, Ann. of Math. 78 (1963), 267-288.

[21] E. Hairer, C. Lubich, G. Wanner, Geometric numerical integration - Structurepreserving algorithms for ordinary differential equations, Springer Series in Computational Mathematics, vol. 31 (2002), Springer-Verlag, Berlin.

[22] J.-L. Loday, Cup-product for Leibniz cohomology and dual Leibniz algebras, Math. Scand. 77 no. 2 (1995), 189-196. 
[23] J.-L. Loday, Dialgebras, Lect. Notes Math. 1763, Springer, Berlin (2001), pp. 7-66.

[24] J.-L. Loday, M Ronco, Combinatorial Hopf algebras, Preprint arXiv:0810.0435v1 [math.QA] (2008).

[25] W. Magnus, On the exponential solution of differential equations for a linear operator, Commun. Pure Appl. Math. 7, (1954) 649-673.

[26] A. Murua, The Hopf algebra of rooted trees, free Lie algebras, and Lie series, Found. Comput. Math., DOI 10.1007/s10208-003-0111-0 (2006), 387-426.

[27] D. Manchon, A. Saïdi, Lois pré-Lie in interaction, Preprint arXiv:math/0811.2153 (2009).

[28] M. P. Schützenberger, Sur une propriété combinatoire des algèbres de Lie libres pouvant être utilisée dans un problème de mathématiques appliquées, Séminaire DubreilJacotin Pisot (Algèbre et théorie des nombres), Paris, Année 1958/59.

[29] E. B. Vinberg, The theory of homogeneous convex cones, Transl. Moscow Math. Soc. 12 (1963), 340-403.

Dominique Manchon, Laboratoire de Mathématiques, CNRS-UMR6620, Université Blaise Pascal, 24 Avenue des Landais, Les Cézeaux, BP 80026, F63177 Aubière CEDEX, France

E-mail: manchon@math.univ-bpclermont.fr 